\title{
Nuevas evidencias en Insuficiencia Cardiaca con Ivabradina
}

\author{
New evidences in heart failure with ivabradine
}

\author{
Alfonso Varela Román \\ Servicio de Cardiología. Complejo Hospitalario Universitario de Santiago. Hospital Clínico de Santiago de Compostela
}

\section{Introducción}

El tratamiento de la insuficiencia cardiaca (IC) con función sistólica deprimida es uno de los campos de la medicina cardiovascular en donde se han producido mayores avances en las últimas décadas, permitiendo reducir de forma significativa su elevada mortalidad y morbilidad. El tratamiento farmacológico basado en el bloqueo neurohormonal ha demostrado aumentar la supervivencia, reducir hospitalizaciones y mejorar la capacidad funcional y calidad de vida de los pacientes que sufren esta enfermedad ${ }^{(1)}$. Sin embargo han pasado muchos años sin que hubiese novedades relevantes en este campo. La ivabradina, cuyo efecto farmacológico principal y casi exclusivo es la reducción de la frecuencia cardiaca (FC), surge, avalada por el estudio SHIFT (2), como una nueva alternativa en el tratamiento de este grupo de pacientes.

Múltiples evidencias nos sugieren que existe una relación directa entre la FC y el riesgo cardiovascular. Estudios realizados en población general, sin enfermedad cardiovascular conocida, sugieren una relación directa entre FC en reposo y mortalidad total por cualquier causa y muerte súbita por infarto de miocardio ${ }^{(3)}$. Resultados similares se han observado también en pacientes con cardiopatía isquémica crónica, como en el registro Coronary Artery Surgery Study, en el que en más de 20.000 pacientes, se observó una relación directa entre la
FC en reposo y la mortalidad total y cardiovascular, que se mantenía después de ajustar por otros factores de riesgo(4). La reducción de la FC en reposo con betabloqueantes o con fármacos como la ivabradina que no modifica la actividad neurohormonal, es capaza de limitar la disfunción sistólica ventricular observada en modelos experimentales de IC en animales(5). Los estudios con betabloqueantes en pacientes con infarto agudo de miocardio e IC sugieren que la reducción observada en la mortalidad es, al menos en parte, debida a la reducción de la FC obtenida con estos fármacos. Kjekshus analizó la relación entre el tratamiento, la FC y la mortalidad en estudios de pacientes con IC y observó una relación entre el grado de reducción de la FC y la mortalidad, observando incluso un incremento en la mortalidad cuando la FC en reposo aumentaba ${ }^{(6)}$. En un metanálisis de 23 ensayos clínicos con betabloqueantes en IC, la magnitud de la reducción de la FC obtenida y no la dosis de betabloqueantes se relacionaba con la reducción en la mortalidad, de forma que por cada 5 latidos de reducción en la FC en reposo, la mortalidad se reducía en un $18 \%$, tal como podemos observar en la figura $1^{(7)}$.

Parece pues que existe una relación entre FC en reposo y el pronóstico en pacientes con IC. Los betabloqueantes han demostrado mejorar la supervivencia en estos pacientes, y este

Figura 1. Relación entre reducción de FC y mortalidad en metanálisis de 23 ensayos clínicos con betabloqueantes en IC con función sistólica deprimida. Modificado de referencia 7

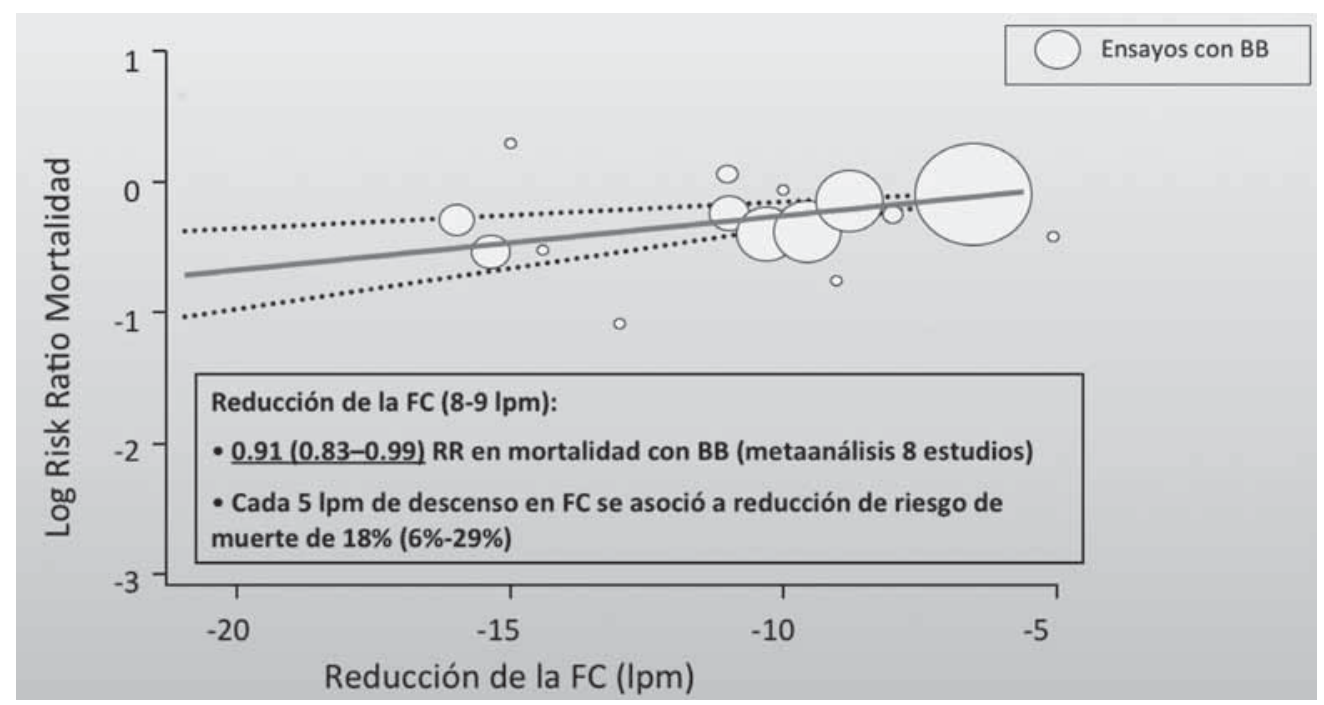


beneficio parece explicarse al menos en parte por la reducción en la FC en reposo. A pesar de ello existe una proporción significativa de pacientes con IC tratados con betabloqueantes que presentan valores altos de FC en reposo. Este es el escenario en el que se planteó el estudio SHIFT con ivabradina ${ }^{(2)}$. La ivabradina es un fármaco que bloquea específicamente la corriente If de las células marcapasos del nodo sinusal reduciendo la FC en reposo. En dosis terapéuticas no actúa sobre otros receptores cardiacos ni vasculares y a diferencia de los betabloqueantes no modifica la contractilidad miocárdica, ni la conducción intracardiaca. El estudio SHIFT fue un ensayo clínico randomizado, doble ciego, con grupos paralelos, en pacientes con IC sintomática son fracción de eyección ventricular izquierda (FEVI) de 35\% o menor, en ritmo sinusal con FC de 70 latidos por minuto o mayor, hospitalizados por IC en el último año, y en tratamiento estándar, incluyendo betabloqueante si eran tolerados. Se randomizaron 6.558 pacientes y el objetivo primario del estudio fue un combinado de muerte de causa cardiovascular u hospitalización por empeoramiento de insuficiencia cardiaca. Tras un seguimiento medio de 22,9 meses el $24 \%$ de los pacientes del grupo ivabradina y $29 \%$ del grupo placebo, presentaron un evento del objetivo primario (HR 0,82, 95\% Cl 0,75-0,90, p < 0,0001). Esta reducción en el objetivo primario fue ya evidente desde el tercer mes de tratamiento. Los beneficios obtenidos en el grupo ivabradina se concentraron en los objetivos relacionados con insuficiencia cardiaca (muerte por IC, hospitalización por agravamiento de IC), tal como podemos ver en la figura 2. La mortalidad por cualquier causa fue ligeramente inferior en el grupo de ivabradina, aunque estas diferencias no fueron significativas. Los resultados del estudio SHIFT aclaran ciertas cuestiones sobre la relación entre FC y pronóstico en IC y sientan la indicación de ivabradina en pacientes con IC con las características de los incluidos en el ensayo clínico. Pero como suele suceder en investigación estos resultadas plantean nuevas cuestiones, a alguna de las cuales se ha intentado dar respuesta en diferentes subestudios publicados recientemente y que intentaremos repasar en esta revisión.

\section{Reducción de la frecuencia cardiaca con ivabradina y calidad de vida en pacientes con insuficiencia cardiaca}

La IC crónica condiciona una importante merma en la calidad de vida de los pacientes que la sufren, existiendo una relación directa entre el grado de deterioro de la calidad de vida y un peor pronóstico en términos de morbilidad y mortalidad (8). La evaluación de la calidad de vida es importante en pacientes con IC ya que la reducción de los síntomas y la mejora en la calidad de vida son objetivos primarios del tratamiento. El subestudio de calidad de vida del SHIFT pretende evaluar si la reducción de la FC conseguida con ivabradina en pacientes con IC se traduce en una mejora en la calidad de vida de los pacientes y si ésta se asocia a una reducción en los eventos primarios del estudio ${ }^{(9)}$. En 1.944 pacientes, 968 en el grupo ivabradina y 976 en el grupo placebo, se evaluó la calidad de

Figura 2: Reducción de eventos y nivel de significación estadística, observada con ivabradina en el estudio SHIFT. Modificada de referencia 2. Hosp: hospitalización

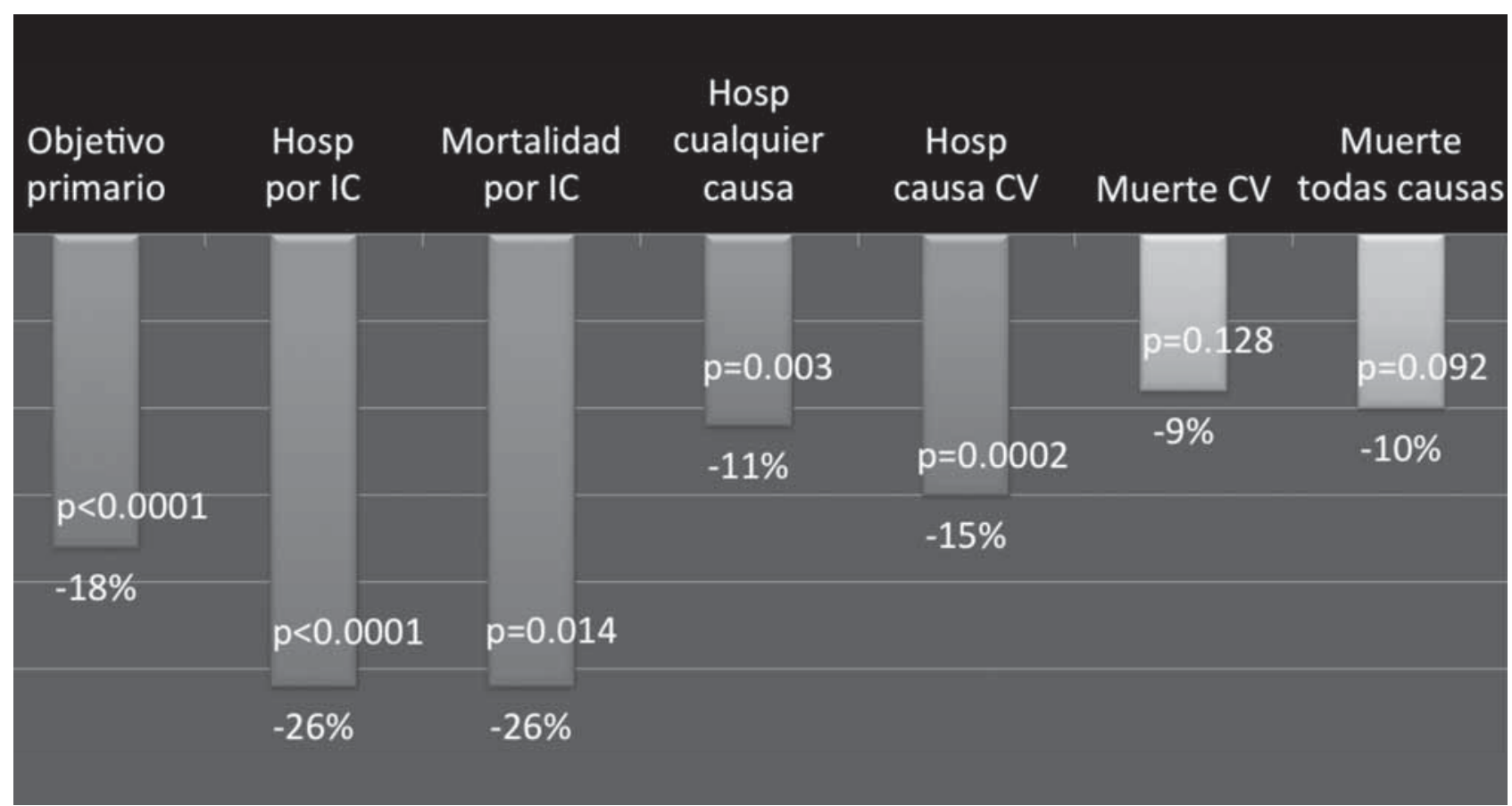


vida mediante el cuestionario de miocardiopatía de Kansas City, basalmente y en diferentes periodos del seguimiento (4 meses, 12 meses, 24 meses y tras última visita). Al primer año de seguimiento los pacientes con puntuaciones más bajas en el test de calidad de vida presentaban un mayor número de eventos del objetivo primario, tal como puede verse en la figura 3. Tras 12 meses de seguimiento los pacientes del grupo tratado con ivabradina presentaron una mayor reducción de la frecuencia cardiaca que los del grupo placebo, con una

Figura 3: Objetivo compuesto primario en función de puntación global en cuestionario de miocardiopatías de Kansas City. Modificado de referencia 9

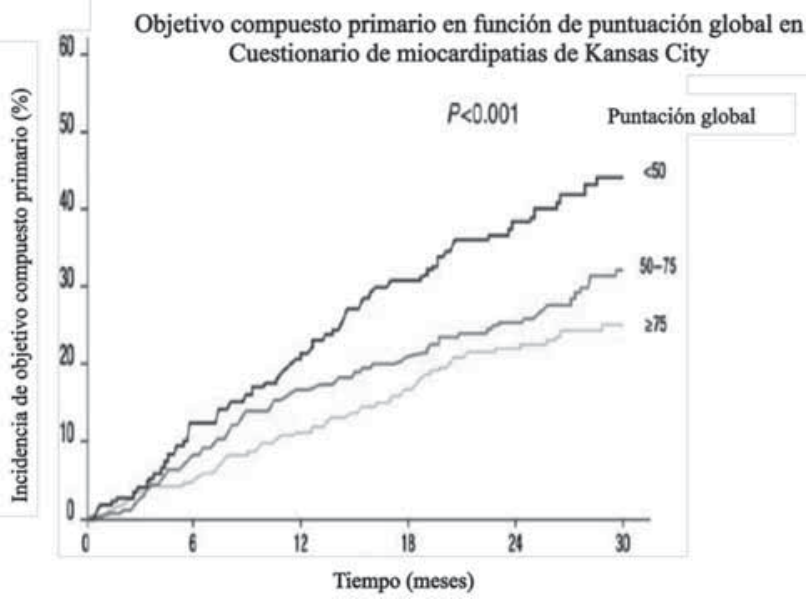

test de calidad de vida, aunque esta fue mayor en el grupo de ivabradina, tal como puede verse en la figura 4. Estos datos confirman que existe una relación inversa entre la FC en reposo y la calidad de vida en pacientes con IC sistólica y además que la reducción de esta con ivabradina mejora la puntación en el cuestionario de calidad de vida, de forma paralela a la reducción en los objetivos primarios del estudio. La IC presenta tasas elevadas de mortalidad y morbilidad y por tanto los objetivos primarios del tratamiento deben ser la reducción de la mortalidad y las hospitalizaciones. Sin embargo, la mortalidad refleja de una manera pobre la forma en la que el paciente percibe su enfermedad en el día a día, de ahí la importancia de disponer de estrategias terapéuticas que reduzcan la mortalidad y la morbilidad, pero que también tengan un impacto positivo sobre la calidad de vida y la sintomatología del paciente.

\section{Efecto de la reducción de la FC con ivabradina sobre el remodelado y la función ventricular izquierda}

El remodelado cardiaco juega un papel central en la fisiopatología de la IC, de forma que la dilatación y la reducción de la FEVI constituyen dos potentes predictores de un pronóstico diferencia de -10,1 Ipm (intervalo confianza 95\%: -11.1 a -9,9; $p<0,0001$ ), y un mayor incremento en la puntación del cuestionario de calidad de vida, tanto en la puntación clínica, con una diferencia de 1,8 puntos (intervalo confianza 95\%: 0,3 a 3,2; p < 0,018), como en la puntación global, con una diferencia a favor de ivabradina de 2,4 puntos (intervalo confianza 95\%: 0,9 a 3,9; $p<0,001)$. En ambos grupos se observó una relación significativa entre el grado de reducción de la frecuencia cardiaca y la mejoría en la puntuación en el

Figura 4: relación entre los cambios en la FC tras 12 primeros meses y cambios en puntuación global en el cuestionario de calidad de vida, en el grupo de ivabradina y en el grupo placebo. Modificado de referencia 9

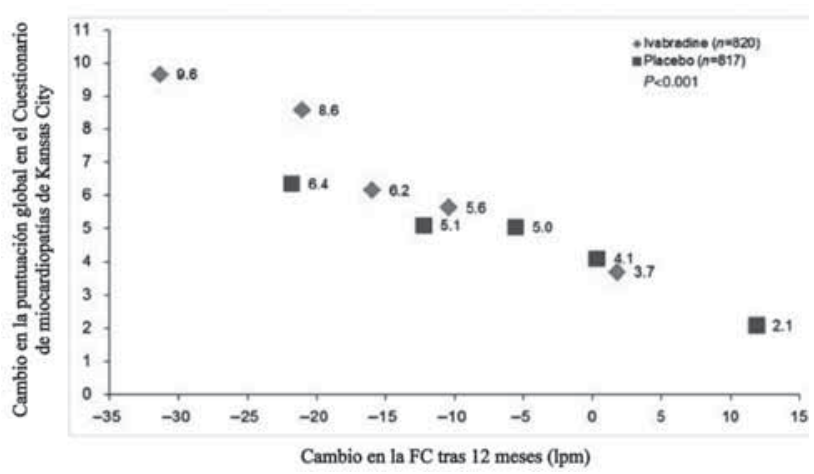

desfavorable en estos pacientes. Diferentes estrategias terapéuticas indicadas en IC sistólica, como betabloqueantes e inhibidores del enzima de conversión de angiotensina (IECA) y también no farmacológicas como la terapia de resincronización cardiaca, han relacionado sus beneficios pronósticos con mejoría en el remodelado cardiaco ${ }^{(10)}$. Un reciente metanálisis publicado por Kramer, en el que se incluyen 30 ensayos clínicos de mortalidad, con 25 fármacos/dispositivos y 88 estudios de remodelado con el mismo fármaco o dispositivo, nos muestra que existe una relación entre el efecto en el remodelado a corto plazo de estas estrategias terapéuticas, sobre la FEVI y los volúmenes ventriculares y la reducción en la mortalidad a largo plazo(10). El aumento en el valor de la FEVI, tal como puede observarse en la figura 5, o la reducción en los volúmenes diastólico o sistólico ventriculares izquierdos, se relacionan con una menor mortalidad a largo plazo.

En 411 pacientes del estudio SHIFT, 208 del grupo ivabradina y 203 del grupo placebo, se llevó a cabo un subestudio ecocardigráfico, con un ecocardiograma basal y a los 8 meses de seguimiento, con objeto analizar el efecto de ivabradina sobre el remodelado ventricular en pacientes con IC sistólica ${ }^{(11)}$. Los pacientes con ventrículos izquierdos basalmente más dilatados, con un índice de volumen telesistólico ventricular izqui- 
Figura 5: relación entre el cambio en la FEVI y la mortalidad en ensayos clínicos controlados con placebo. Modificado de referencia 10

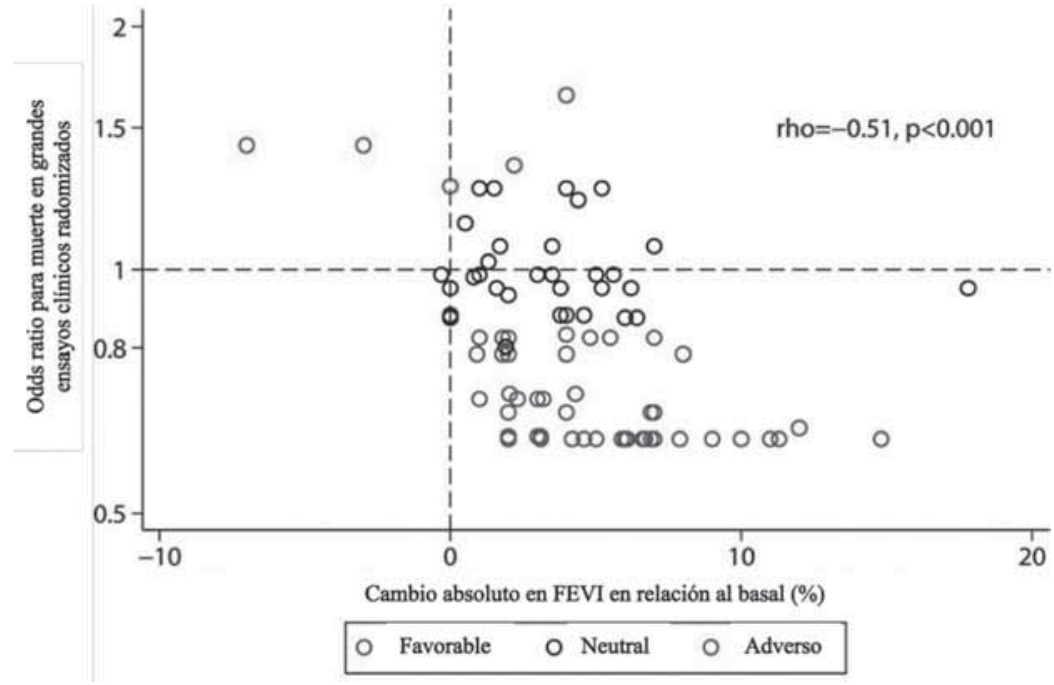

erdo superior a la media $(59 \mathrm{ml} / \mathrm{m} 2)$, presentaron un peor pronóstico, con una mayor incidencia de eventos del objetivo primario compuesto (Hazard Ratio 1.62, intervalo de confianza 95\%: 1,03-2,56, $p=0.04)$. Tras 8 meses de tratamiento, en el grupo tratado con ivabradina, se observó un remodelado más favorable que en el grupo placebo, con una mayor reducción de los índices de volumen telesistólico (- 7,0 \pm 16,3 vs. $-0,9 \pm 17,1 \mathrm{ml} / \mathrm{m} 2 ; p<0,001)$ y telediastólico ventricular izquierdo (- 7,9 $\pm 18,9$ vs. $-1,8 \pm 19,0 \mathrm{ml} / \mathrm{m} 2, p=0,002)$, así como un mayor incremento en la FEVI $(+2,4 \pm 7,7$ vs. $0,1 \pm 8,0 \%, p<0,001)$. Estos cambios fueron independientes del tratamiento con betabloqueantes y la etiología de la IC. Este remodelado más favorable, con mayores reducciones en el índice de volumen telesistólico ventricular izquierdo, se asoció con un mejor pronóstico, con una menor incidencia de eventos del objetivo primario. Se observó también una relación inversa entre la reducción de la FC y la mejoría en la FEVI $(r=-0,17, p=0.0006)$, presentando un mayor incremento en la FEVI aquellos que presentaron una mayor reducción en la FC en relación a la basal. La respuesta del remodelado al tratamiento en el estudio no fue homogénea en todos los pacientes, aunque hubo una mayor proporción de pacientes en el grupo tratado con ivabradina que presentaron un remodelado favorable, tal como podemos ver en la figura 6 . Este hecho es destacable ya que más de un tercio de los pacientes del grupo ivabradina presentaron un incremento en la FEVI superior al $5 \%$, mientras que esta respuesta no alcanzo a la cuarta parte del grupo placebo. Este incremento significativo en la FEVI puede tener implicaciones terapéuticas, ya que la FEVI es un parámetro muy importante a la hora de establecer indicaciones terapéuticas con desfibriladores implantables 0 con marcapasos con terapia de resincronización ${ }^{(12)}$.

Los resultados obtenidos en este subestudio son importantes ya que el remodelado cardiaco juega un papel central en la progresión de la IC. El tratamiento con ivabradina invierte el remodelado ventricular en pacientes con IC sistólica, y lo consigue a pesar de que más del 90\% recibía tratamiento con betabloqueantes y antagonistas del eje renina-angiotensina, grupos farmacológicos que han demostrado ser capaces de mejorar el remodelado cardiaco en este grupo de pacientes. Los datos sugieren una conexión entre la mejoría en el remodelado cardiaco conseguida con ivabradina y el pronóstico, siendo estos resultados consistentes con los del metanálisis publicado por Kramer en los que se observa que mejorías a corto plazo en el remodelado cardiaco se acompañan de reducciones en la mortalidad a largo plazo(10).

\section{Influencia de la dosis de betabloqueantes en los resultados del estudio SHIFT}

El estudio SHIFT se realizo en pacientes que recibían tratamiento farmacológico de acuerdo a las guías de práctica clínica, de forma que en torno al $90 \%$ de los pacientes recibían tratamiento con betabloqueantes y antagonistas del sistema renina-angiotensina. La dosis de betabloqueante fue inferior a la utilizada en los ensayos clínicos de betabloqueantes en IC, pero superior a la dosis media reportada en registros que recogen la práctica clínica habitual(13). El 26\% de los pacientes recibía la dosis de betabloqueantes recomendada en las guías de práctica clínica y el $56 \%$ al menos el $50 \%$ de esta dosis. La hipotensión y la fatiga fueron los principales motivos que limitaron la utilización de dosis mayores de betabloqueantes. En el grupo de pacientes tratados con al menos el $50 \%$ de la dosis máxima recomendada en las guías, no se observó reducción significativa en el objetivo primario del estudio, objetivando tan solo una reducción significativa en la hospitalización por IC, de un 19\%. Es de destacar que la incidencia anual de eventos del objetivo primario en este subgrupo de pacientes fue claramente inferior al observado en el grupo total, un 13\% frente a un 18\%. Este hecho puede explicar la 
Figura 6: remodelado ventricular, con cambio relativo en índice de volumen telesistólico ventricular izquierdo (IVTSVI) y FEVI tras 8 meses de tratamiento, en grupo ivabradina y grupo placebo. Modificado de referencia 11

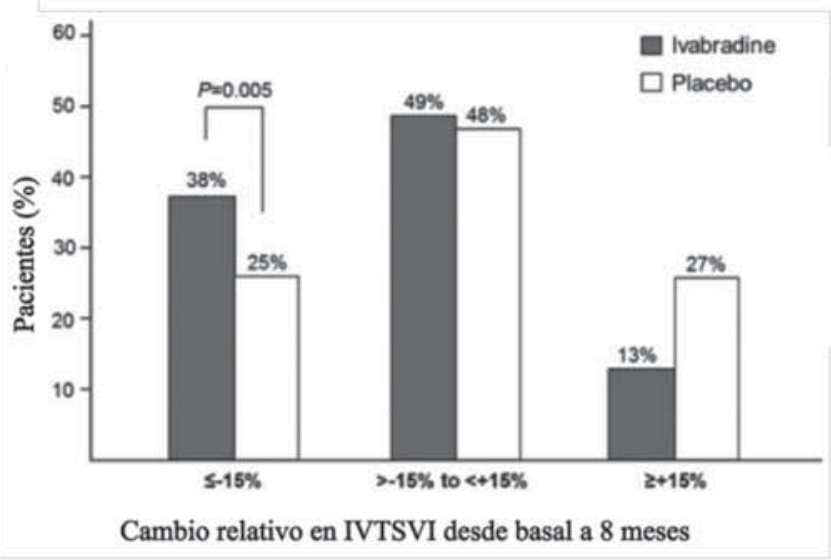

ausencia de diferencias estadísticamente significativas. Aunque no hubo interacción en el efecto de la ivabradina entre los grupos de pacientes que tomaban y que no tomaban betabloqueantes al inicio del ensayo clínico, una cuestión que quedo pendiente en el SHIFT fue si la dosis de betabloqueante utilizada pudo haber influido en los resultados. Este fue el motivo de la realización de un subestudio en el que se analizaron los resultados en función de la dosis de betabloqueante ${ }^{(14)}$. Los subgrupos de pacientes en función de la dosis de betabloqueantes presentaban diferencias significativas en cuanto a sus características clínicas. Factores independientes asociados a baja probabilidad de uso de betabloqueantes son los antecedentes de EPOC, asma bronquial, baja presión arterial, alta FC en reposo, ser mayor o recibir tratamiento con amiodarona, calcioantagonistas o digoxina, y los de baja

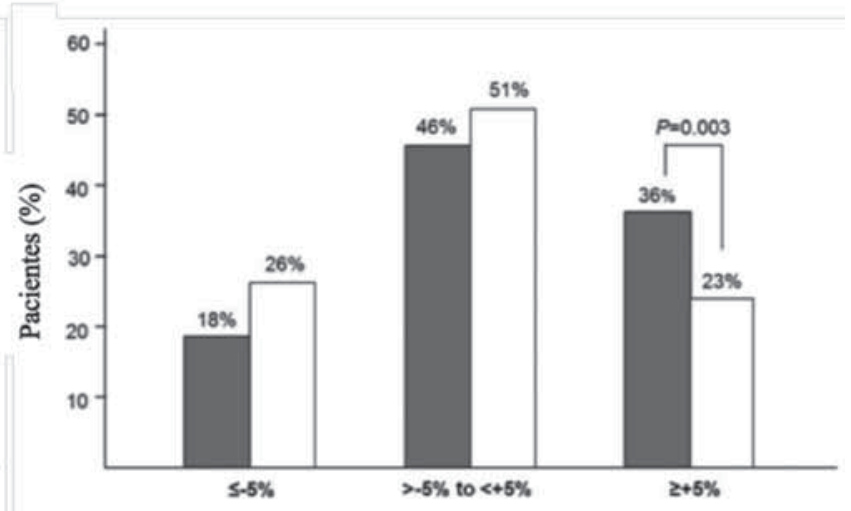

Cambio absoluto en FEVI desde basal a 8 meses

probabilidad de utilización de al menos el $50 \%$ de la dosis recomendada en las guías son antecedentes de EPOC, baja presión arterial, alta frecuencia cardiaca en reposo, ser mayor, recibir tratamiento con amiodarona o digoxina, y no recibir tratamiento con calcioantagonistas, tal como puede verse en la tabla 1. Por tanto las diferencias en la respuesta al tratamiento con ivabradina en los diferentes subgrupos de dosis de betabloqueantes pueden estar condicionadas por estas diferencias clínicas. De hecho en los pacientes que reciben al menos el $50 \%$ de la dosis recomendada la incidencia de eventos del objetivo primario es menor que en el grupo total. Otro aspecto reseñable es observar el grado de reducción de la FC a los 28 días de tratamiento, en el grupo de ivabradina en relación con el grupo placebo, en función de la dosis basal de betabloqueantes y de la FC basal, tal como

Tabla 1: Resultados análisis multivariado para identificar factores independientes asociados a baja probabilidad de utilización de betabloqueantes 0 de dosis de betabloqueantes al menos 50\% de las recomendadas por guías de práctica clínica. Modificado de referencia 14

\begin{tabular}{|c|c|c|}
\hline & BB vs no BB & $\begin{array}{c}\geq 50 \% \text { dosis objetivo BB vs }<50 \% \text { dosis } \\
\text { objetivo BB } \\
\text { OR (IC 95\%), valor } p\end{array}$ \\
\hline Historia EPOC & OR (IC 95\%), valor $p$ & $0,67(0,55-0,80),<0,0001$ \\
\hline Historia Asma & $0,23(0,19-0,28),<0,0001$ & $\mathrm{~N} / \mathrm{A}$ \\
\hline PAD, cada 5 mm Hg menor & $0,13(0,10-0,19),<0,0001$ & $\mathrm{~N} / \mathrm{A}$ \\
\hline PAS, cada 5 mm Hg menor & $0,90(0,86-0,95),<0,0001$ & $0,82(0,79-0,85),<0,0001$ \\
\hline FC, cada 5 Ipm mayor & $\mathrm{N} / \mathrm{A}$ & $0,95(0,92-0,97), 0,0002$ \\
\hline Edad, cada 5 años mayor & $0,81(0,78-0,84),<0,0001$ & $0,91(0,89-0,94),<0,0001$ \\
\hline Tratamiento & $0,83(0,80-0,87),<0,0001$ & $0,63(0,44-0,89), 0,0093$ \\
Amiodarona & & $1.33(1.07-1.64), 0.0090$ \\
\hline Calcioantagonistas & $0,29(0,20-0,42),<0,0001$ & $0,75(0,65-0,85),<0,0001$ \\
\hline Digoxina & $0,52(0,40-0,69),<0,0001$ & $0,58(0,48-0,70),<0,0001$ \\
\hline
\end{tabular}

BB: betabloqueante; EPOC: enfermedad pulmonar obstructiva crónica; PAD: presión arterial diastólica; PAS: presión arterial sistólica; FC: frecuencia cardiaca; N/A: no aplicable por no haber sido incluido en el modelo. 
Figura 7: diferencia en el cambio medio de FC entre ivabradina y placebo desde el valor inicial a los 28 días, en cada uno de los 25 subgrupos definidos por la línea de base beta-bloqueante. Modificado de referencia 14

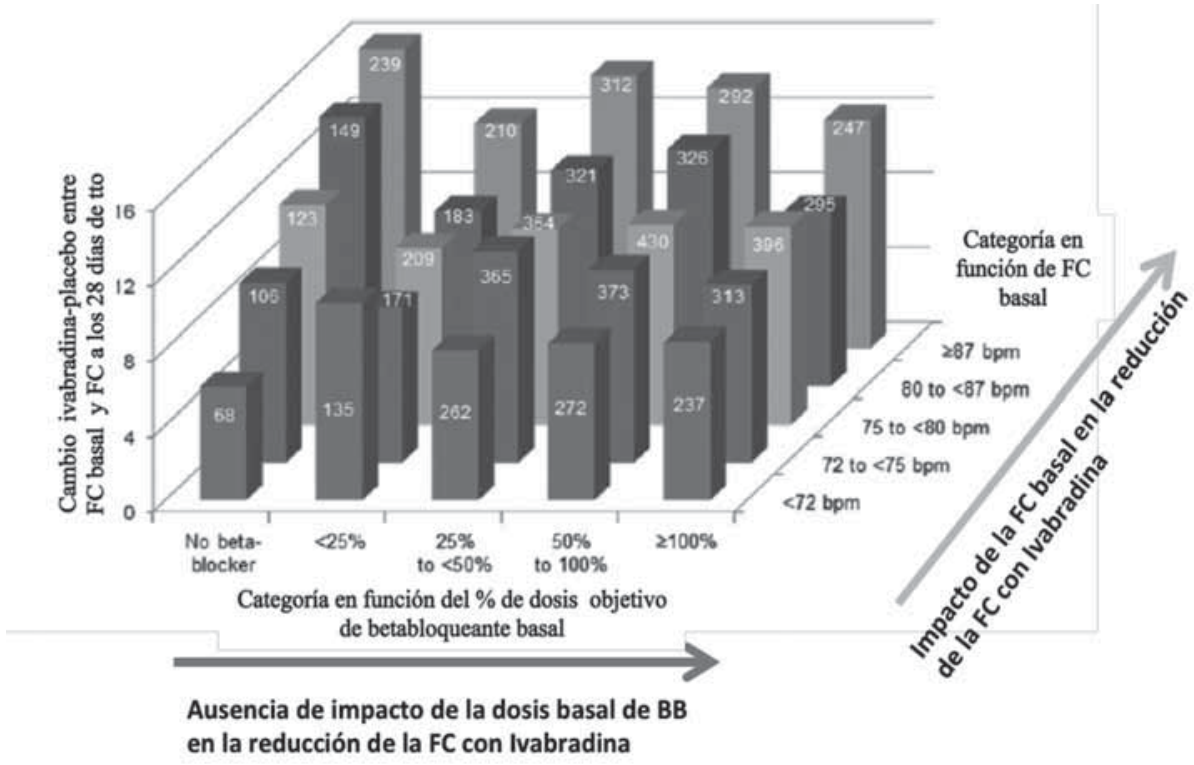

podemos observar en la figura 7. No se observa interacción significativa entre la dosis basal de betabloqueantes y el grado de reducción de FC conseguido con ivabradina. Sin embargo, sí que existe interacción significativa entre el grado de reducción de FC a los 28 días y la FC basal ( $p<0,0001)$. La incidencia del objetivo primario se redujo de forma significativa en todos los subgrupos con dosis de betabloqueantes inferiores al $50 \%$ de la recomendada por las guías de práctica clínica de la Sociedad Europea de Cardiología, incluyendo el grupo sin betabloqueantes, tal como podemos ver en la tabla 2. Aunque se objetiva una tendencia decreciente en el beneficio sobre el objetivo primario a medida que aumenta la dosis de betabloqueantes, la interacción entre los subgrupos no resulta estadísticamente significativa ( $p$ $=0,35$ ). Esta interacción casi alcanza significación estadística cuando se utiliza un test específico que valora la tendencia entre los diferentes grupos de dosis de betabloqueantes $(p=0,056)$, sin embargo cuando se ajusta por la interacción observada entre la FC basal y el grado de reducción de la FC con ivabradina esta débil tendencia desaparece $(p=0,135)$. Estos datos sugieren que es la magnitud en la reducción de la FC conseguida con ivabradina asociada a betabloqueantes, el principal determinante en el resultado del estudio, más que la dosis de betabloqueantes. En pacientes con IC sistólica la dosis máxima de betabloqueantes que puede alcanzarse depende de las características del paciente y de la presencia de comorbilidades. Si tras titular la dosis del betabloqueante la FC basal es inferior a 70 lpm, se puede considerar que el tratamiento es óptimo y no es necesario asociar ivabradina, pero si tras alcanzar la dosis máxima tolerada la FC es superior a 70 lpm, la asociación de ivabradina resultará beneficiosa en términos pronósticos, de manera especial, cuanto mayor sea la FC basal.

Tabla 2: efecto de la ivabradina sobre el objetivo primario del estudio en función de la dosis basal de betabloqueantes. Modificado de referencia 14

\begin{tabular}{|c|c|c|c|c|c|c|}
\hline $\begin{array}{c}\text { Categoría BB } \\
\% \text { dosis objetivo }\end{array}$ & $\begin{array}{c}\text { Tasa eventos } \\
\text { placebo (\%) }\end{array}$ & Hazard ratio & IC 95\% & $\begin{array}{c}p \\
\text { heterog. }\end{array}$ & $\begin{array}{c}p \\
\text { tend. }\end{array}$ & \\
\hline $\begin{array}{c}\text { Objetivo primario } \\
\text { (muerte, hospitaliz. } \\
\text { x IC) }\end{array}$ & & & & & \\
No BB & 39,3 & 0,71 & $0,55-0,93$ & & \\
BB, 25\% & 40 & 0,74 & $0,59-0,92$ & & \\
BB, $25-<50 \%$ & 30,8 & 0,81 & $0,68-0,98$ & & \\
BB, $50-<100 \%$ & 24,8 & 0,88 & $0,72-1,07$ & 0,35 & 0,056 & \\
BB, $\geq 100 \%$ & 20,1 & 0,99 & $0,79-1,24$ & & \\
\hline
\end{tabular}

BB: betabloqueante; IC 95\%: intervalo de confianza 95\%; p heterog.: nivel significación test heterogeneidad; p tend.: nivel significación del test de tendencia entre las categorías de dosis de betabloqueantes; p tend. aj.: nivel de significación del test de tendencia ajustado para FC basal y la interacción entre FC basal y el tratamiento asignado. 


\section{Influencia de la FC basal en los resultados del estudio SHIFT}

Los resultados del estudio SHIFT nos muestran que la FC basal es no solo un marcador de riesgo en pacientes con IC sistólica, sino también un factor de riesgo modificable. En un análisis detallado del papel de la FC en el estudio SHIFT se observó que la FC basal era un potente predictor de eventos en el seguimiento, de forma que cada incremento de $5 \mathrm{lpm}$ en la FC basal suponía un aumento en la incidencia del objetivo primario combinado de muerte cardiovascular u hospitalización por IC del 15,6\% ${ }^{(15)}$. En este mismo análisis se observa que el riesgo de hospitalización por IC aumenta ya con FC superiores a 70 lpm, sin embargo el umbral a partir del cual aumenta la mortalidad cardiovascular, se sitúa en torno a los 75 Ipm $^{(15)}$. Por otra parte el análisis de los resultados del SHIFT en los subgrupos preespecificados fue consistente en todos ellos, con excepción del análisis en función de la FC basal, en donde se observó un mayor beneficio en el subgrupo con FC superior a la media, $77 \mathrm{lpm}(\mathrm{p}=0,029)$ (2). Basándose posiblemente en estas observaciones la Agencia Europea del Medicamento, aprobó ivabradina para el tratamiento de IC crónica, en clase II a IV de la NYHA, con disfunción sistólica, en pacientes en ritmo sinusal y cuya FC es $\geq 75 \mathrm{Ipm}$, en combinación con terapia estándar incluyendo betabloqueantes 0 cuando los betabloqueantes están contraindicados o no son tolerados. El que no existan datos sobre este grupo de pacientes en los que actualmente la ivabradina está indicada, ha llevado a que se realice un análisis en profundidad de los resultados en el subgrupo de pacientes con FC basal $\geq 75 \mathrm{lpm}$, que se ha publicado recientemente ${ }^{(16)}$. En el análisis de la influencia de la FC en el estudio SHIFT se observó que el mayor beneficio se obtenía en los pacientes que presentaban una FC basal más alta, pero también en aquellos en los que tras optimizar la dosis de ivabradina se obtenía un mayor grado de reducción de la FC y se conseguía una FC más baja. Por este motivo en este subestudio se analizó también la FC alcanzada y el grado de reducción de la misma y su influencia en los resultados.

Los 4.150 pacientes del estudio SHIFT con FC basal $\geq 75$ Ipm, presentaban características clínicas diferentes a los 2.351 con $\mathrm{FC}<75$ Ipm. Los primeros eran más jóvenes, presentaban una mayor proporción de fumadores, una FEVI menor, una clase NYHA mayor y una etiología no isquémica en mayor proporción. La utilización de betabloqueantes en el grupo de FC $\geq 75 \mathrm{lpm}$ fue en menor, aunque el porcentaje en dosis óptima 0 al menos el $50 \%$ de ésta fue similar. En este grupo el tratamiento con digoxina era más frecuente. Estas diferencias clínicas también se observaron en el pronóstico, siendo más desfavorable en el subgrupo de FC $\geq 75 \mathrm{lpm}$, así los tratados con placebo en este subgrupo presentaron una tasa de eventos del objetivo primario del $33 \%$, frente al $21 \%$ en los tratados con placebo del subgrupo de $\mathrm{FC}<75 \mathrm{lpm}$. Es importante tener en cuenta que esta menor incidencia de eventos en el subgrupo de $\mathrm{FC}<75 \mathrm{lpm}$ puede condicionar la ausencia de beneficios en este subgrupo. En el subgrupo de FC $\geq 75 \mathrm{lpm}$, el tratamiento con ivabradina reduce el objetivo primario un 24\% (HR 0,76, IC $95 \%$ Cl 0,68-0,85, p<0,0001), la mortalidad por cualquier causa un $17 \%$ (HR 0,83, IC $95 \%$, $0,72-0,96, p=0,0109$ ), la mortalidad cardiovascular un $17 \%$ (HR 0,83, IC $95 \%, 0,71-0,97, p=0.0166$ ), la mortalidad por IC un 39\% (HR 0,61, IC $95 \%, 0,46-0,81, p=0.0006)$, la hospitalizaciones por IC un 30\% (HR 0,70, IC $95 \%, 0,61-$ $0,80, p<0,0001$ ), las hospitalizaciones por cualquier causa un $18 \% \%$ (HR 0,82, IC $95 \%, 0,75-0,90, p<0,0001$ ) y las hospitalizaciones de causa cardiovascular un $21 \%$ (HR 0,79,

Tabla 3: efecto del tratamiento con ivabradina sobre pronóstico en el subgrupo de pacientes con FC $\geq 75$ Ipm (4.150 pacientes). Modificado de referencia 16

\begin{tabular}{|c|c|c|c|c|}
\hline & $\begin{array}{c}\text { Tasa eventos (\%) } \\
\text { Ivabradina }\end{array}$ & $\begin{array}{c}\text { Tasa eventos (\%) } \\
\text { Placebo }\end{array}$ & $\begin{array}{c}\text { Hazar Ratio } \\
\text { (IC 95\%) }\end{array}$ & Valor p \\
\hline $\begin{array}{c}\text { Objetivo primario } \\
\text { Muerte CV u Hospitaliz. XIC }\end{array}$ & $27 \%$ & $33 \%$ & $0,76(0,68-0,85)$ & $<0,0001$ \\
\hline Mortalidad & & & & \\
Cualquier causa & $17 \%$ & $19 \%$ & $0,83(0,72-0,96)$ & 0,012 \\
CV & $15 \%$ & $17 \%$ & $0,83(0,71-0,97)$ & 0,017 \\
IC & $4 \%$ & $6 \%$ & $0,61(0,46-0,81)$ & $<0,0001$ \\
\hline Hospitalizaciones & $18 \%$ & $24 \%$ & $0,70(0,61-0,86)$ & $<0,0001$ \\
IC & $39 \%$ & $44 \%$ & $0,82(0,75-0,90)$ & $<0,0001$ \\
Cualquier causa & $31 \%$ & $37 \%$ & $0,79(0,71-0,88)$ & $<0,0001$ \\
CV & & & \\
\hline
\end{tabular}

CV: cardiovascular; IC: insuficiencia cardiaca. 
IC $95 \%, 0,71-0,88, p<0,0001)$, tal como puede observarse en la tabla 3. En el subgrupo de FC $<75$ Ipm, no se observó reducción significativa del objetivo primario ni de ninguno de estos objetivos secundarios. En el subgrupo de FC $\geq 75 \mathrm{lpm}$ el grado de reducción de riesgo con ivabradina depende de la FC alcanzada a los 28 días de tratamiento, siendo mayor el beneficio cuando la reducción de la FC es $\geq 10$ Ipm o cuando la FC alcanzada es $<60$ lpm. En el subgrupo de FC $<75$ Ipm se observa una tendencia favorable a ivabradina en términos de reducción de hospitalizaciones por IC y mortalidad por IC, cuando la reducción de la FC es $\geq 10 \mathrm{lpm} 0$ cuando la FC alcanzada es $<60 \mathrm{lpm}$. Por tanto el beneficio conseguido tras optimización de dosis de ivabradina parece depender la FC alcanzada y del grado de reducción, así el mayor beneficio se

Figura 8: incidencia anual de eventos del objetivo primario en el grupo tratado con ivabradina, en función del grado de reducción de la FC y de la FC alcanzada a los 28 días de tratamiento. Los pacientes que presentaron un evento del objetivo primario en los primeros 28 días fueron excluidos

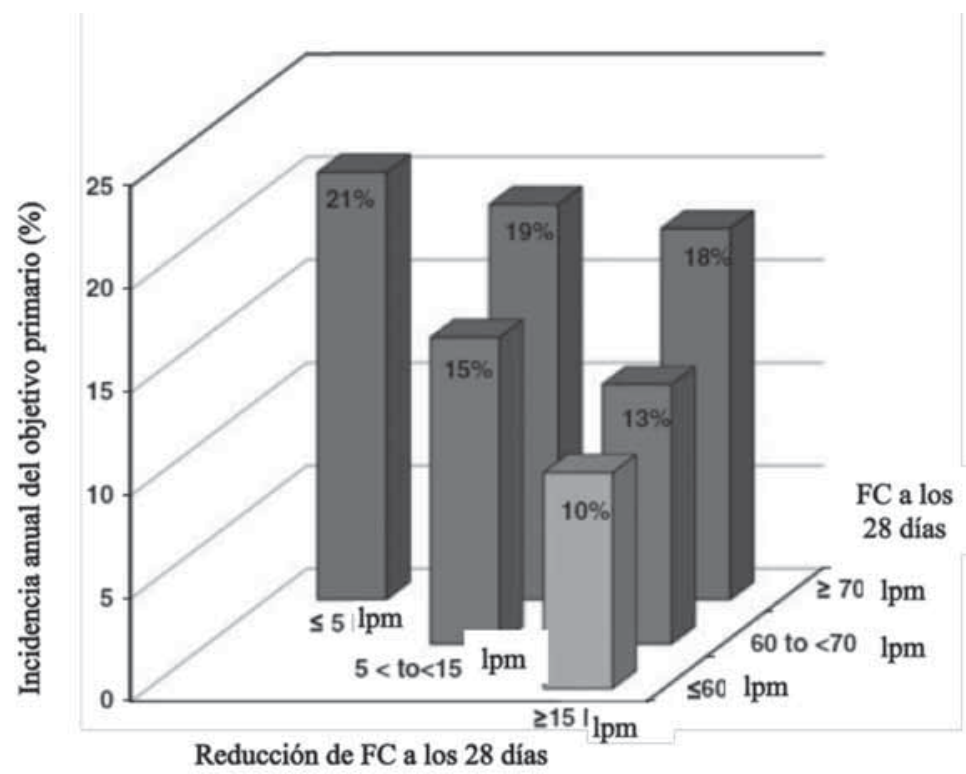

observa en aquellos pacientes en los que la FC se reduce más de $10 \mathrm{lpm}$ y es inferior a $60 \mathrm{lpm}$, tal como puede verse en la figura 8. Otro aspecto reseñable de este subestudio es que la tolerancia de ivabradina no parece depender la FC basal, ya que no se observan diferencias significativas en los efectos adversos entre los subgrupos de $\mathrm{FC} \geq 75 \mathrm{Ipm}$ y $\mathrm{FC}<75 \mathrm{Ipm}$. El tratamiento con ivabradina reduce eventos cardiovasculares desfavorables en pacientes con IC sistólica y FC elevada, cuando se administra asociada a los fármacos recomendados por las guías de práctica clínica. Este efecto es especialmente pronunciado cuando se administra a pacientes con $\mathrm{FC} \geq 75$ Ipm, y cuando tras optimizar la dosis se consigue reducir la FC más de 10 lpm y se alcanzar una FC inferior a 60 lpm.

\section{Efectos de ivabradina en pacientes tratados con antialdosterónicos}

Hasta su última revisión, las guías de práctica clínica en IC recomendaban la utilización de antagonistas de los receptores de aldosterona en pacientes con IC sistólica en estadios avanzados, en clase III y IV de la NYHA (17). Esta indicación se basaba fundamentalmente en los resultados del estudio RALES, realizado con espironolactona en pacien- tes con IC sistólica en clase funcional III y IV de la NYHA ${ }^{(18)}$. Sin embargo la publicación de los resultados del estudio EMPHASIS-HF, que comparó eplerenona con placebo, en IC sistólica en clase funcional no tan avanzada, ha hecho que la últimas guías de práctica clínica en IC amplíen la indicación de estos fármacos, asociados a IECAs y betabloqueantes, a todos los pacientes con IC sintomática, con FEVI $\leq 35 \%{ }^{(12)}$. Aunque la utilización de este tipo de fármacos en IC sistólica ya era amplia este cambio en las guías previsiblemente hará que aumente su porcentaje de utilización. Por este motivo los investigadores del estudio SHIFT analizaron los resultados con ivabradina en el subgrupo de pacientes tratados con antagonistas de aldosterona ${ }^{(20)}$.

De los 6.505 pacientes del estudio SHIFT, un 60\%, 3.922 pacientes recibían tratamiento con antagonistas de los receptores de la aldosterona, en su gran mayoría con espironolactona, 96\%. Las características clínicas de estos pacientes eran diferentes a las de los 2.583 que no los recibían. Los pacientes tratados con estos fármacos eran más jóvenes, presentaban una clase funcional más avanzada, NYHA III-IV, en mayor proporción, los antecedentes de HTA, cardiopatía isquémica e infarto de miocardio eran menos frecuentes y sus 
Tabla 4: características clínicas en pacientes tratados y no tratados con antagonistas de la aldosterona. Modificado de referencia 20

\begin{tabular}{|c|c|c|c|}
\hline & $\begin{array}{c}\text { Antialdosteronicos } \\
3.922 \text { pacientes }\end{array}$ & No Antialdosterónicos & Valor $p$ \\
\hline Edad media (años) & 59 & 6.583 & $<0,0001$ \\
\hline Etiología isquémica & $63 \%$ & $76 \%$ & $<0,0001$ \\
\hline NYHA II & $45 \%$ & $54 \%$ & $<0,0001$ \\
\hline NYHA III & $53 \%$ & $45 \%$ & $<0,0001$ \\
\hline FEVI \% & $28 \%$ & $30 \%$ & $<0,0001$ \\
\hline FC (Ipm) & 80 & 79 & $<0,0001$ \\
\hline PAS (mm Hg) & 119 & 126 & $<0,0001$ \\
\hline PAD (mm Hg) & 75 & 77 & \\
\hline
\end{tabular}

cifras de presión arterial y FEVI eran también menores. La FC era ligeramente superior en este grupo, y no había diferencias significativas en el filtrado glomerular estimado. En relación al tratamiento era más frecuente en este grupo la utilización de diuréticos y digoxina. Estas características podemos verlas en la tabla 4. Estas diferencias clínicas condicionan el que la incidencia de eventos del objetivo primario sea mayor en los pacientes tratados con placebo en el subgrupo tratado con antagonistas de los receptores de aldosterona, con una incidencia de un $33 \%$ frente a un $23 \%$ en el subgrupo no tratado con estos fármacos. A pesar de estas diferencias al analizar el objetivo combinado primario, sus componentes individuales y otros objetivos secundarios, no se objetiva interacción significativa en función del tratamiento con antagonistas de la aldosterona, tal como puede verse en la figura 9. La magnitud de la reducción del objetivo primario (muerte cardiovascular u hospitalización por IC), así como la observada en otros eventos, como la muerte cardiovascular, la hospitalización por IC, la mortalidad por IC y la mortalidad por cualquier causa, fue similar en el grupo tratado con estos fármacos y en el grupo que no los recibía. Tampoco se objetivaron diferencias significativas de seguridad entre ambos grupos.

Con las limitaciones de tratarse de un estudio no preespecificado sus resultados nos sugieren que el beneficio clínico del tratamiento con ivabradina, en pacientes con IC sistólica, en ritmo sinusal, con FC basal $\geq 70 \mathrm{lpm}$, tratados de acuerdo con las guías de práctica clínica, es similar en pacientes que reciben o no tratamiento con antagonistas de la aldosterona.

Figura 9: resultados de objetivo primario y otros objetivos secundarios en pacientes tratados y no tratados con antagonistas de aldosterona. Modificado de referencia 20

\begin{tabular}{|c|c|c|c|c|}
\hline & Ivabradina & Placebo & Hazard ratio & $\mathrm{p}$ interacción \\
\hline \multicolumn{5}{|l|}{ Objetivo primario ( $\%$ ) } \\
\hline Con $\mathrm{AA}, \mathrm{n}=3922$ & 28 & 33 & 0,82 & 0,916 \\
\hline $\operatorname{Sin} A A, n=2583$ & 19 & 23 & 0,81 & \\
\hline \multicolumn{5}{|l|}{ Mortalidad CV (\%) } \\
\hline Con AA & 16 & 18 & 0,88 & 0,279 \\
\hline $\operatorname{Sin} \mathrm{AA}$ & 11 & 11 & 1,02 & \\
\hline \multicolumn{5}{|c|}{ Hospitalización por IC (\%) } \\
\hline $\operatorname{Sin} \mathrm{AA}$ & 19 & 23 & 0,77 & 0,304 \\
\hline Con AA & 11 & 17 & 0,67 & \\
\hline \multicolumn{5}{|l|}{ Mortalidad total (\%) } \\
\hline Con AA & 17 & 19 & 0,88 & 0,366 \\
\hline $\operatorname{Sin} \mathrm{AA}$ & 13 & 13 & 0,99 & \\
\hline \multicolumn{5}{|l|}{ Mortalidad por IC ( $\%)$} \\
\hline Con AA & 4 & 6 & 0,73 & 0,723 \\
\hline $\operatorname{Sin} \mathrm{AA}$ & 3 & 3 & $0,80_{\text {Favorable Ivabradina }}$ & Favorable Placebo \\
\hline
\end{tabular}




\section{Ivabradina en las guías de práctica clínica de la Sociedad Europea de Cardiología}

Las evidencias científicas procedentes del estudio SHIFT han motivado el que el tratamiento con ivabradina, en pacientes con IC sistólica sea contemplado en las últimas guías de práctica clínica de la Sociedad Europea de Cardiología. A diferencia de la indicación aprobada por la Agencia Europea del Medicamente, que la establece para una $F C \geq 75 \mathrm{lpm}$, las guías indican el fármaco en base a los criterios de inclusión del estudio SHIFT y por lo tanto consideran una $\mathrm{FC} \geq 70 \mathrm{lpm}$. Las nuevas guías establecen dos indicaciones, una como recomendación de clase lla y nivel de evidencia B, en la que se recomienda el tratamiento con ivabradina para prevenir las hospitalizaciones por IC, en pacientes en ritmo sinusal, con FEVI $\leq 35 \%, F C \geq 70$ Ipm, que persisten sintomáticos (NYHA II-IV) a pesar de tratamiento en dosis basadas en la evidencia de betabloqueantes (o máximas toleradas si son menores a las recomendadas), IECAs (0 antagonistas de los receptores de angiotensina II) y antagonistas de la aldosterona. La segunda indicación es una recomendación de clase llb, con nivel de evidencia $\mathrm{C}$, en la que se considera el tratamiento para pacientes en ritmo sinusal, con $\mathrm{FEVI} \leq 35 \%$, que no toleran betabloqueantes, debiendo recibir tratamiento con IECAs (o antagonistas de los receptores de angiotensina II) y antagonistas de la aldosterona. Aunque el nivel de recomendación no es de clase I, en el flujograma de tratamiento recomendado por las guías, la ivabradina aparece recomendado en el $4^{\circ}$ escalón, para tratar pacientes con FEVI $\leq 35 \%$, en ritmo sinusal, con $\mathrm{FC} \geq 70 \mathrm{lpm}$, que persisten sintomáticos una vez titulados hasta las dosis recomendadas Ios IECA (o antagonistas de receptor de angiotensina II si éstos no son tolerados), los betabloqueantes y los antagonistas del receptor de aldosterona.

Sin entrar en la cuestión de la FC basal a partir de la cual debe estar indicada la ivabradina, lo que parece quedar claro es que la evidencia sugiere de forma consistente que el tratamiento con ivabradina mejora de forma significativa el pronóstico en pacientes con IC sistólica, en ritmo sinusal y FC elevada, que persisten sintomáticos tras optimización de tratamiento con antagonistas del sistema renina-angiotensina, betabloqueantes y antagonistas de aldosterona, y que este beneficio potencial parece mayor cuanto mayor sea la FC basal.

\section{Bibliografía}

1. Norgard NB, Stark JE. Pharmacotherapy for heart failure with left ventricular dysfunction: beyond angiotensin-converting enzyme inhibitors and beta-blockers. Pharmacotherapy. 2008; 28: 920-31.

2. Swedberg K, Komajda M, Böhm M, Borer JS, Ford I, Dubost-Brama A, et al. Ivabradine and outcomes in chronic heart failure (SHIFT): a randomized placebo-controlled study. Lancet. 2010; 376: 875-85.

3. Jouven X, Empana JP, Schwartz PJ, Desnos M, Courbon D, Ducimetière P. Heart-rate profile during exercise as a predictor of sudden death. N Engl J Med. 2005; 352: 1951-8.

4. Diaz A, Bourassa MG, Guertin MC, Tardif JC. Long-term prognostic value of resting heart rate in patients with suspected or proven coronary artery disease. Eur Heart J. 2005; 26: 967-74.

5. Fox K, Borer JS, Camm AJ, Danchin N, Ferrari R, Lopez Sendon JL, et al. Heart Rate Working Group. Resting heart rate in cardiovascular disease. J Am Coll Cardiol. 2007; 50: 823-30.

6. Kjekshus J, Gullestad L. Heart rate as a therapeutic target in heart failure. Eur Heart J Suppl 1999; 1 Suppl H: H64-9.

7. McAlister FA, Wiebe N, Ezekowitz JA, Leung AA, Armstrong PW. Meta-analysis: betablocker dose, heart rate reduction, and death in patients with heart failure. Ann Intern Med. 2009; 150: 784-94.

8. Juenger J, Schellberg D, Kraemer S, Haunstetter A, Zugck C, Herzog W, et al. Health related quality of life in patients with congestive heart failure: comparison with other chronic diseases and relation to functional variables. Heart. 2002; 87: 235-41.

9. Ekman I, Chassany 0, Komajda M, Böhm M, Borer JS, Ford I, et al. Heart rate reduction with ivabradine and health related quality of life in patients with chronic heart failure: results from the SHIFT study. Eur Heart J. 2011; 32: 2395-404.

10. Kramer DG, Trikalinos TA, Kent DM, Antonopoulos GV, Konstam MA, Udelson JE. Quantitative evaluation of drug or device effects on ventricular remodeling as predictors of therapeutic effects on mortality in patients with heart failure and reduced ejection fraction: a meta-analytic approach. J Am Coll Cardiol. 2010; 56: 392-406.

11. Tardif JC, O'Meara E, Komajda M, Böhm M, Borer JS, Ford I, et al. Effects of selective heart rate reduction with ivabradine on left ventricular remodelling and function: results from the SHIFT echocardiography substudy. Eur Heart J. 2011; 32: 2507-15.

12. McMurray JJ, Adamopoulos S, Anker SD, Auricchio A, Böhm M, Dickstein K, et al. ESC Guidelines for the diagnosis and treatment of acute and chronic heart failure 2012: The Task Force for the Diagnosis and Treatment of Acute and Chronic Heart Failure 2012 of the European Society of Cardiology. Developed in collaboration with the Heart Failure Association (HFA) of the ESC. Eur J Heart Fail. 2012; 14: 803-69.

13. de Groote $P$, Isnard R, Clerson $P$, Jondeau G, Galinier M, Assyag P, et al. Improvement in the management of chronic heart failure since the publication of the updated guidelines of the European Society of Cardiology. The Impact-Reco Programme. Eur $\mathrm{J}$ Heart Fail. 2009; 11: 85-91.

14. Swedberg K, Komajda M, Böhm M, Borer J, Robertson M, Tavazzi L, et al. Effects on outcomes of heart rate reduction by ivabradine in patients with congestive heart failure: is there an influence of beta-blocker dose?: findings from the SHIFT (Systolic Heart failure treatment with the I(f) inhibitor ivabradine Trial) study. J Am Coll Cardiol. 2012; 59: 1938-45

15. Böhm M, Swedberg K, Komajda M, Borer JS, Ford I, Dubost-Brama A, et al. Heart rate as a risk factor in chronic heart failure (SHIFT): the association between heart rate and outcomes in a randomised placebo-controlled trial. Lancet. 2010; 376: 886-94.

16. Böhm M, Borer J, Ford I, Gonzalez-Juanatey JR, Komajda M, Lopez-Sendon J, et al. Heart rate at baseline influences the effect of ivabradine on cardiovascular outcomes in chronic heart failure: analysis from the SHIFT study. Clin Res Cardiol. 2012 May 11. [Epub ahead of print].

17. Dickstein K, Cohen-Solal A, Filippatos G, McMurray JJ, Ponikowski P, Poole-Wilson $P A$, et al. ESC Guidelines for the diagnosis and treatment of acute and chronic heart failure 2008: the Task Force for the Diagnosis and Treatment of Acute and Chronic Heart Failure 2008 of the European Society of Cardiology. Developed in collaboration with the Heart Failure Association of the ESC (HFA) and endorsed by the European Society of Intensive Care Medicine (ESICM). Eur Heart J. 2008; 29: 2388-442.

18. Pitt B, Zannad F, Remme WJ, Cody R, Castaigne A, Perez A, et al. The effect of spironolactone on morbidity and mortality in patients with severe heart failure. Randomized Aldactone Evaluation Study Investigators. N Engl J Med. 1999; 341: 709-17.

19. Zannad F, McMurray JJ, Krum H, Van Veldhuisen DJ, Swedberg K, Shi H, et al. Eplerenone in patients with systolic heart failure and mild symptoms. N Engl J Med $2011 ; 364: 11-21$

20. Komajda M, Böhm M, Borer J, Ford I, Krum H, Tase A, et al. Influence of background treatment with mineralocorticoid receptor antagonists on ivabradine's effects in patients with chronic heart failure. Eur J Heart Fail. 2012 Aug 14. [Epub ahead of print]. 